\title{
Homeostatic Plasticity Mediated by Rod-Cone Gap Junction Coupling in Retinal Degenerative Dystrophic RCS Rats
}

\section{OPEN ACCESS}

Edited by:

Davide Cervia,

Tuscia University, Italy

Reviewed by:

Bela Volgyi

University of Pécs, Hungary

Giovanni Casini,

University of Pisa, Italy

*Correspondence:

CongJian Zhao

cj.zhao@yahoo.com

Zheng Qin Yin

ginzyin@aliyun.com

${ }^{\dagger}$ These authors have contributed equally to this work.

Received: 10 January 2017 Accepted: 22 March 2017

Published: 20 April 2017

Citation:

Hou B, Fu Y, Weng C, Liu W, Zhao C and Yin ZQ (2017) Homeostatic Plasticity Mediated by Rod-Cone Gap Junction Coupling in Retinal Degenerative Dystrophic RCS Rats.

Front. Cell. Neurosci. 11:98. doi: 10.3389/fncel.2017.00098

\author{
Baoke Hou ${ }^{1,2+}$, Yan Fu ${ }^{1,3 \dagger}$, Chuanhuang Weng ${ }^{1,3}$, Weiping Liu ${ }^{1,3}$, Congjian Zhao ${ }^{1,3 *}$ and \\ Zheng Qin Yin ${ }^{1,3 *}$ \\ ${ }^{1}$ Southwest Hospital/Southwest Eye Hospital, Third Military Medical University, Chongqing, China, ${ }^{2}$ Department of \\ Ophthalmology, Chinese PLA General Hospital, Beijing, China, ${ }^{3}$ Key Lab of Visual Damage and Regeneration and \\ Restoration of Chongqing, Chongqing, China
}

Rod-cone gap junctions open at night to allow rod signals to pass to cones and activate the cone-bipolar pathway. This enhances the ability to detect large, dim objects at night. This electrical synaptic switch is governed by the circadian clock and represents a novel form of homeostatic plasticity that regulates retinal excitability according to network activity. We used tracer labeling and ERG recording in the retinae of control and retinal degenerative dystrophic RCS rats. We found that in the control animals, rod-cone gap junction coupling was regulated by the circadian clock via the modulation of the phosphorylation of the melatonin synthetic enzyme arylalkylamine $\mathrm{N}$-acetyltransferase (AANAT). However, in dystrophic RCS rats, AANAT was constitutively phosphorylated, causing rod-cone gap junctions to remain open. A further b/a-wave ratio analysis revealed that dystrophic RCS rats had stronger synaptic strength between photoreceptors and bipolar cells, possibly because rod-cone gap junctions remained open. This was despite the fact that a decrease was observed in the amplitude of both a- and b-waves as a result of the progressive loss of rods during early degenerative stages. These results suggest that electric synaptic strength is increased during the day to allow cone signals to pass to the remaining rods and to be propagated to rod bipolar cells, thereby partially compensating for the weak visual input caused by the loss of rods.

Keywords: gap junctions, retinal pigmentosa, synaptic plasticity, melatonin, AANAT

\section{INTRODUCTION}

The retinal circadian clock governs the extent and strength of rod-cone gap junction coupling in the retinae of vertebrates, including macaques, mice, goldfish, and zebrafish (Ribelayga et al., 2002; Völgyi et al., 2013; Li et al., 2014). This functional switch allows cones to receive very dim light signals from rods at night but not during the day (Ribelayga et al., 2008). The process of increasing rod-cone gap junction coupling may provide vertebrates with the ability to detect large, dim objects. The mechanism underlying the regulation of rod-cone synapses resembles homeostatic plasticity but involves electrical synapses and circadian clock-regulated effects on the compensatory shaping of rods (Curti and O'Brien, 2016).

The Royal College of Surgeons (RCS) rat is a good animal model for studying the early onset of retinal degenerative diseases (Sauvé et al., 2001). In this rat, a substantial loss of rod photoreceptors 
occurs several months before the loss of cones, and the combination of these events results in eventual blindness (Girman et al., 2005). Retinal degeneration is initiated in this animal model during late retinal synaptic functional maturation (Van Den Berghe et al., 2004). The synaptic connections and synaptic strength between photoreceptors and bipolar cells are still being refined during late maturation, after the eyes have opened. In RCS mice, a malfunctional RPE results in rod cell death (D'Orazi et al., 2014). Gap junctions but not rod-cone coupling via gap junctions have been observed in rat photoreceptors (Guo et al., 2014). Presumably, during the progressive loss of rods in dystrophic RCS rats, the loss of night vision would be partially compensated if signaling by the remaining rods was capable of reaching the cones via gap junction couplings. Here, we investigated whether gap junction coupling continues to occur in dystrophic RCS rats during the early onset of retinal degeneration. Next, we investigated whether this gap junction-mediated homeostatic plasticity is modulated by the retinal circadian clock. Finally, we investigated the impact of this form of homeostatic plasticity on dystrophic RCS rats. To address these questions, we studied rod-cone gap junction coupling, the mechanism underlying this process, and its functional impact by combining tracer labeling and ERG recording in control and retinal degenerative dystrophic RCS rat retinae.

\section{MATERIALS AND METHODS}

\section{Neural Retina Preparation}

All experimental and animal handling procedures were performed in compliance with the Association for Research in Vision and Ophthalmology Statement and reviewed and approved by the Faculty Committee on the Use of Live Animals in Teaching and Research, Third Military Medical University. RCS (RCS-rdy-) rats and their congenic controls (RCS-rdy+) were used as control groups. Rats in these two groups (aged $\mathrm{P} 10, \mathrm{P} 14, \mathrm{P} 20, \mathrm{P} 25$, and P35) were tested for the ratio of gap junction coupling between the neurons in the outer plexiform layer (OPL) such as rod, cone. The animals were housed with a $12 \mathrm{~h}$ light $-12 \mathrm{~h}$ dark cycle (with lights on at 6 A.M. and off at 6 P.M.). The experimental procedures were conducted in constant darkness $(\leq 0.0001$ lux $)$. The experimenters using infrared night-vision goggles to visualize the experiments. RCS and control rats were deeply anesthetized using a mixture of ketamine $(70 \mathrm{mg} / \mathrm{kg})$ and xylazine $(7 \mathrm{mg} / \mathrm{kg})$ after a $3 \mathrm{~h}$ dark adaptation period. They were then enucleated, and the anterior of each eyeball was removed and placed into an extracellular bath solution that was continuously bubbled with 95\% $\mathrm{O}_{2}-5 \%$ $\mathrm{CO}_{2}$ and had the following composition per liter (1): $8.8 \mathrm{~g}$ of Ames' Medium (Sigma; Cat\# A1420), $1.9 \mathrm{~g}$ of $\mathrm{NaHCO}_{3}$, and 7 $\mathrm{mL}$ of penicillin-streptomycin (Sigma; Cat\# P0781). Osmolarity was then adjusted to $\sim 280 \mathrm{mOsm}$. The retina was placed flat on the front of a piece of filter paper (Millipore, Cat\#AABP02500) with the photoreceptor side up.

\section{Cut-Loading}

The protocol for cut-loading was modified from previous reports (Ribelayga et al., 2008; Choi et al., 2012). Briefly, the retinae were incubated in Ames' media containing bicarbonate and bubbled with $95 \% \mathrm{O}_{2}-5 \% \mathrm{CO}_{2}$ in 6-well-plates for $30 \mathrm{~min}$ in the dark, as described above, with, or without test drugs. In this study, the retinae were separated among the six following groups: daytime (noon) without any drugs, nighttime (midnight) without any drugs, melatonin agonist during the daytime (2Iodomelatonin, Tocris Cat. \#0737; Delete "during the daytime"), melatonin antagonist (Luzindole, Tocris Cat. \#0877) during the nighttime, melatonin receptor $(\mathrm{MT} 1,2)$ agonist (melatonin, Tocris Cat. \#3550) during the daytime, MT2 antagonist (4-PPDOT, Tocris Cat. \#1034) during the nighttime (Delete D1/D2 antagonists). We used the dyes Lucifer Yellow (0.5\%, Sigma, Cat\# L0259-25 MG, MW 457.25) and dextran tetramethylrhodamine (0.5\%, Invitrogen, Cat\# D-1817, MW 10,000) as the tracer solutions. A filter paper with a retina attached was placed on a glass slide so that the neural retina was exposed. Then, a razor blade was dipped in the tracer solution and used to make a radial cut through the retina and filter paper. The blade was held perpendicular to the retina and filter. This procedure was repeated until four cuts were made per retina. Next, filter paper was attached to the top of the neural retina, and the retina was then moved into a bubbled extracellular bath solution. After the retina was incubated for $15 \mathrm{~min}$ at $35^{\circ} \mathrm{C}$, it was washed three times for 5 min each in a new bubbled extracellular solution (with or without drugs, depending on the first incubation conditions). The tissues were then fixed in 2\% paraformaldehyde/1.5\% glutaraldehyde in $0.1 \mathrm{M}$ phosphate buffer (PBS, pH 7.4) for $1 \mathrm{~h}$ at RT(3). The retinae were washed with $0.1 \mathrm{M}$ PBS overnight at $4^{\circ} \mathrm{C}$ and then detached from the filter paper using PBS and placed onto a slide with the photoreceptor side facing the glass.

\section{Imaging}

After the retinae were mounted in VectaShield mounting medium, the slides were immediately observed and imaged using an Olympus laser-scanning confocal microscope. Images were recorded at a resolution of $512 \times 512$ pixels $(635.90 \times 635.90$ microns) at a depth of 44 microns. The images were reloaded using Fiji software, and $\mathrm{Z}$ projections were used to evaluate the fluorescence of the outer neural retina or the OPL.

\section{Immunostaining}

Enucleated eyecups were immersed in PBS containing 4\% paraformaldehyde at $4^{\circ} \mathrm{C}$ for $2 \mathrm{~h}$ and then incubated in $30 \%$ sucrose at $4^{\circ} \mathrm{C}$ overnight. The eyecups were embedded in O.C.T. compound and cut into $10 \mu \mathrm{m}$-thick sections. The sections were permeabilized using $0.5 \%$ Triton $\mathrm{X}-100$ for $15 \mathrm{~min}$ and then blocked in $5 \%$ goat serum for $1 \mathrm{~h}$. Primary antibodies against AANAT (1:1,000, ab3505, Abcam), p-AANAT (phospho T29, $1: 1,000$, ab3439, Abcam) were diluted in blocking buffer, and the samples were incubated in this solution at $4^{\circ} \mathrm{C}$ overnight. The sections were rinsed three times ( $15 \mathrm{~min}$ each) with PBS at room temperature and then incubated at $37^{\circ} \mathrm{C}$ for $1 \mathrm{~h}$ with secondary antibodies. Cell nuclei were stained using 4,6diamidino-2-phenylindole (DAPI; Invitrogen), and fluorescence images were acquired using a confocal microscope (Zeiss LSM 700, Carl Zeiss) with the same imaging parameters. 


\section{Western Blot}

To perform Western blot analysis of whole retinae, the retinal samples were first lysed in ice-cold RIPA lysis buffer containing protease inhibitors (Beyotime). The lysates were centrifuged at $15,000 \mathrm{~g}$ at $4{ }^{\circ} \mathrm{C}$ for $5 \mathrm{~min}$, and the supernatants were then collected. Protein concentrations were determined using a BCA protein assay (Beyotime). A total of $25 \mu \mathrm{g}$ of protein from each sample was loaded and electrophoresed on an SDS-polyacrylamide gradient gel (SDS-PAGE), and the blots were then transferred to PVDF membranes. The membranes were blocked in $5 \%$ non-fat milk at $37^{\circ} \mathrm{C}$ for $30 \mathrm{~min}$. The membranes were incubated with anti-AANAT (1:1,000, ab3505, Abcam), anti-p-AANAT (phospho T29,1:1,000, ab3439, Abcam) and anti-GAPDH (1:1,000, CW0100, CWBIO) antibodies at $4^{\circ} \mathrm{C}$ overnight. The membranes were then rinsed with TBST and incubated with a horseradish peroxidase (HRP)-conjugated secondary antibody (Beyotime, $1: 1,000$ ) at $37^{\circ} \mathrm{C}$ for $1 \mathrm{~h}$. The blotted proteins were visualized using an ECL detection system (Thermo Scientific).

\section{Electroretinogram (ERG)}

Dark-adapted animals were placed under dim red light to prepare them for recording. Anesthesia was performed as described (Weymouth and Vingrys, 2008). The pupils were dilated by placing one drop each of tropicamide and phenylephrine into the eye. Corneal ERG responses were simultaneously recorded from both eyes using gold wire loops. The reference electrode was inserted under the scleral conjunctiva, and the ground electrode was placed in the tail. Amplification, stimulus presentation, and data acquisition were performed using a Reti-scan system (Roland, Germany). The following stimulus intensities were delivered at an inter-flash interval of $120 \mathrm{~s}:-40,-35,-30,-25$, $-20,-15,-10,-5,0 \mathrm{db}(0.0003,0.00095,0.003,0.0095,0.03$, $0.095,0.3,0.95$, and $3.0 \mathrm{~cd} \cdot \mathrm{s} \cdot \mathrm{m}^{-2}$, respectively).

\section{Data Analysis}

The cut-loading images were rotated so that the dye perfused from left to right, and the cut lines were vertical. Rectangular ROIs were chosen to cover the perfusion areas. ROI of OPL peak mainly contains photoreceptor and horizontal cells (Supplement Figure 2). We used the Plot Profile function in Fiji to obtain the gray value distance curve, and the curves were transformed for use in Igor Pro software. Using Igor Pro 6.0 (WaveMetrics Inc.), the relative fluorescence intensity was obtained by dividing each raw fluorescence value by the maximum fluorescence value, and these data were used to draw the relative fluorescence intensity distance curves. The curve fittings were created using the equation $Y=Y_{0}+Y_{\max }{ }^{*} \exp ^{(-x / \lambda)}$, where $Y$ is the relative fluorescence intensity, $Y_{0}$ is the background fluorescence, $Y_{\max }$ is the maximum relative fluorescence, $\lambda$ is the space (length) constant, and $x$ is the distance from the cut (Ribelayga et al., 2008; Choi et al., 2012). The space constant $(\lambda)$ values were compared among groups and times using $t$-tests or ANOVA.

The ERG data were transferred to Igor Pro 6.2 (WaveMetrics Inc.) for further analysis. For demonstration purposes, the amplitudes of the a-waves were plotted inversely. First, the response-intensity was fitted using the following hyperbolic function (Fulton and Rushton, 1978; Hood and Birch, 1992):

$$
f(V)=V_{\max }{ }^{*} I(I+S),
$$

where $V$ and $V_{\max }$ are amplitudes that were measured using a light stimulus of intensity $I$ or a super-saturating stimulus, respectively, and $S$ is the semi-saturation constant, which is the stimulus intensity needed to elicit a response of half-maximal amplitude. The ratio of the b-wave to the a-wave (b/a-wave ratio) was calculated using raw data and fitting data.

\section{RESULTS}

\section{Modulation of Rod-Cone Gap Junction Coupling in Dystrophic RCS Rats}

To measure gap junction coupling in photoreceptors, Lucifer Yellow (MW 457.25), and dextran tetramethylrhodamine (MW $10,000)$ were loaded during the daytime (noon) and nighttime (midnight, constant darkness) following $1 \mathrm{~h}$ of dark adaptation along the cut edge of flat-mounted retinae obtained from both control and dystrophic RCS rats. The red dye dextran tetramethylrhodamine is a large molecule and was used to display the cut line, whereas the small molecule Lucifer Yellow can diffuse through opened gap junctions and was used to visualize coupled cells (Supplement Figure 1C). In the control rats, the diffusion constants at P14 were $11.0590 \pm 3.439$ (day) (Choi et al., 2012) and $32.054 \pm 4.290$ (night), at P20 were $10.62 \pm 2.108$ (day) and $33.980 \pm 4.765$ (night), and at P35 were $15.8910 \pm 3.485$ (day) and $42.840 \pm 4.703$ (night). Gap junction coupling in rat photoreceptor cells is modulated by the circadian clock (Supplement Figure 1A); this was confirmed by injection of Lucifer Yellow into single photoreceptor under patching pipette (Supplement Figure 1B) which is consistent with previous results in other species (Zhang and $\mathrm{Wu}, 2004$ ). To our surprise, we found that in retinal degeneration dystrophic RCS rats, gap junctions remained open during both day and night. In these rats, the diffusion constants at P14 were 30.360 \pm 4.540 (day) and $21.986 \pm 4.170$ (night), at P20 were 49.210 \pm 4.78 (day) and $41.465 \pm 5.640$ (night), and at P35 were $21.686 \pm 3.245$ (day) and $28.980 \pm 4.548$ (night) (Figure 1). For rod-cone coupling dominates in photoreceptor cells (Ribelayga et al., 2008), we calculate the diffusion in the OPL as rodcone gap junction coupling. Thus, in this rat model, rod-cone gap junctions are functionally coupled during both daytime and nighttime, suggesting that rod-cone gap junction coupling is not regulated by the circadian clock in dystrophic RCS rats.

\section{Phosphorylation of the Melatonin Synthesis Enzyme AANAT Is Regulated by the Circadian Clock}

Melatonin synthesis occurs in a rhythmic pattern that modulates rod-cone gap junction coupling (Day et al., 2010; Sandu et al., 2011; Hiragaki et al., 2014), and this process is controlled by the rate-limiting enzyme arylalkylamine $\mathrm{N}$-acetyltransferase (AANAT; Sakamoto and Ishida, 1998; Pozdeyev et al., 2006; Kashiwagi et al., 2013). AANAT is activated by PKC-mediated 


\section{A}

Day time

\section{Night time}

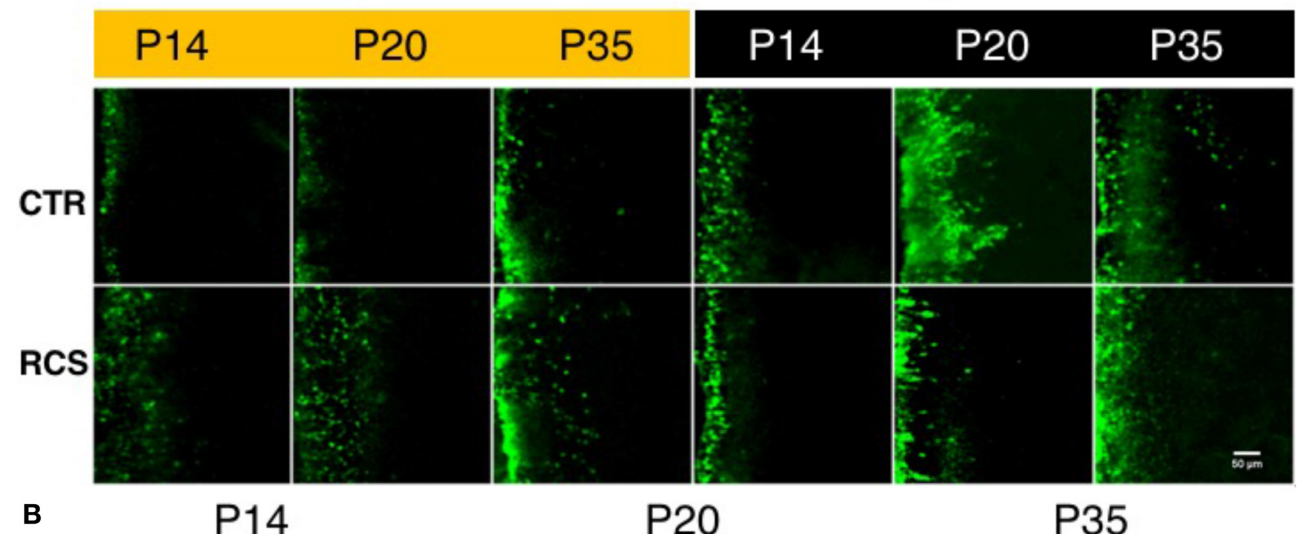

B
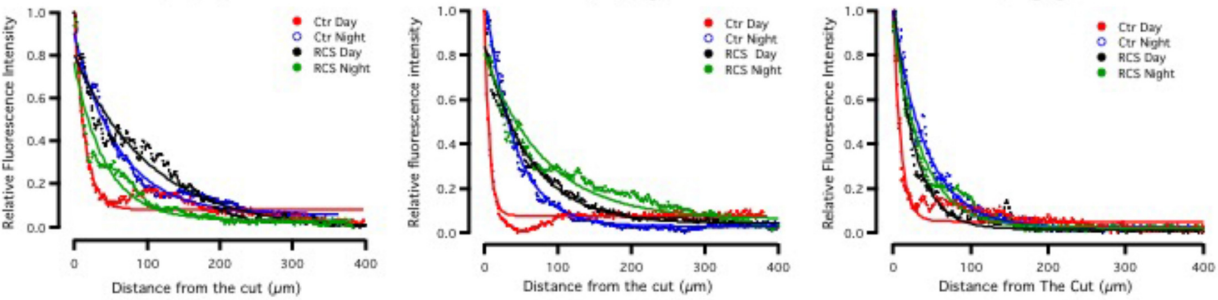

C

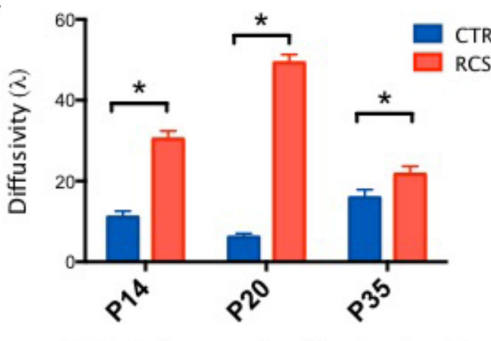

Diffusivity constant to day $(n=6)$

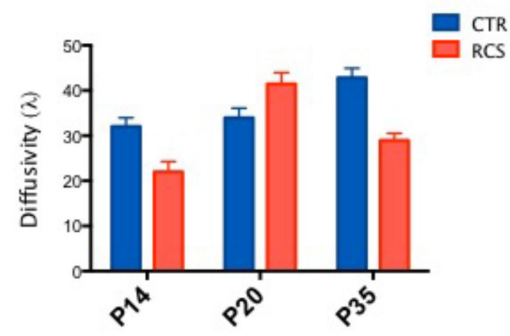

Diffusivity constant to night $(n=6)$

FIGURE 1 | Tracing couplings between photoreceptors in the rat retina. (A) In the control groups, in the outer plexiform layer, the gap junction tracer Lucifer yellow was highly diffused at night (dark label) but not during the day (yellow label) under the same conditions. From P14 to P35, its diffusion capacity increased during both daytime and nighttime in these groups. These data suggest that the gap junction coupling ratio increases at night and decreases during the day and that it increases with age. In dystrophic RCS rats, the diffusion of Lucifer yellow was as extensive at night as during the day and did not increase with age. These data suggest that the gap junction coupling ratio during the daytime and in young pups is higher in dystrophic RCS rats than in controls. (B) The differences in relative fluorescence intensity between the two groups at P14, P20, and P35. The distance in normalized relative fluorescent intensity indicates the gap junction coupling ratio in the OPL in the rat retina. The curvature of the fitting curve (solid line) represents the diffusion capacity under different conditions (the space constant, $\lambda$ ). (C) The difference in the space constant between the daytime and nighttime in control and dystrophic RCS rats. During the daytime, diffusivity was lower in the control group than in the RCS group, and this difference was significant at P14, P20, and P35. In the RCS group, the diffusion capacity during the day increased at P14, was much higher at P20, and then dropped at P35. At night, both the control and RCS groups exhibited high diffusion capacity. The space constant increased over time in the control group but dropped at P35 in the dystrophic RCS rats. In the control rats, the diffusion constants at P14 were $11.0590 \pm 3.439$ (day) and $32.054 \pm 4.290$ (night), at P20 were $6.1062 \pm 2.108$ (day) and $33.980 \pm 4.765$ (night), and at P35 were $15.8910 \pm 3.485$ (day) and $42.840 \pm 4.703$ (night). In contrast, in the dystrophic RCS rats, the diffusion constants at P14 were $30.360 \pm 4.540$ (day) and $21.986 \pm 4.170$ (night), at P20 were $49.210 \pm 4.78$ (day) and $41.465 \pm 5.640$ (night), and at P35 were $21.686 \pm 3.245$ (day) and $28.980 \pm 4.548$ (night). ${ }^{*} P \leq 0.05$

phosphorylation at Thr29 (Choi et al., 2004). To determine the protein expression and distribution patterns of AANAT in the rat retina, we performed immunostaining in the retina using the following two AANAT antibodies: a AANAT antibody and a phosphorylation-specific (pAANAT) antibody. AANAT immunoreactivity was observed at moderate levels in ganglion cells and at low levels in photoreceptor cells (Figure 2A). Western blot analysis showed that the retinae of different groups of rats showed similar AANAT expression levels (Figure 2C). AANAT must be phosphorylated to be activated and to synthesize melatonin. In control rats, pAANAT immunoreactivity was observed in photoreceptor cells at night but not during the day (Figure 2B). In contrast, many strongly pAANATimmunoreactive photoreceptors were observed during both the day and night in dystrophic RCS rats (Figure 2B). These data suggest that in the photoreceptor cells of normal rats, while the 


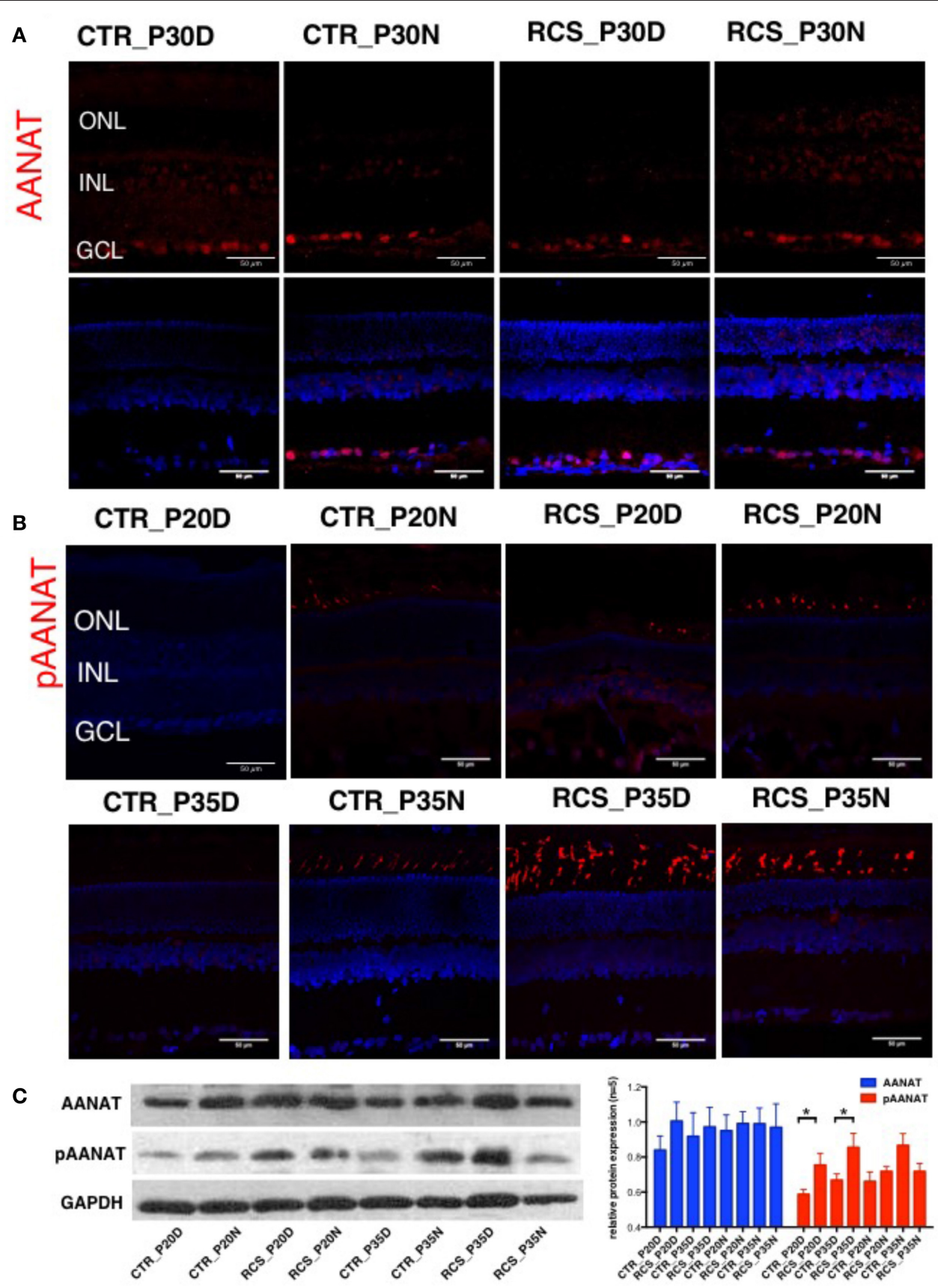

FIGURE 2 | Expression of AANAT and pAANAT in the rats' retina. (A,B) Immunostaining for AANAT and pAANAT in the retinae of control and dystrophic RCS rats. Cell nuclei were stained using DAPI. ONL, outer nuclear layer; INL, inner nuclear layer; GCL, ganglion cell layer. (C) Western blot analysis for pAANAT, AANAT in the retinae of control and dystrophic RCS rats. GAPDH was used as a loading control. The relative protein expression of pAANAT and AANAT is the mean gray value of pAANAT, AANAT/value of GAPDH. Strong relative protein expression was observed in photoreceptor cells at night but not during the day in control rats (B), and high relative protein expression of pAANAT was observed correspondingly (C). Many strongly pAANAT-immunoreactive photoreceptors were observed during both the day and night in dystrophic RCS rats (B), and high pAANAT relative protein expression in $\mathbf{( C )}$. ${ }^{\star} P \leq 0.05$.

total amount of AANAT remains consistent, its phosphorylation is regulated by the circadian clock, allowing the cells to synthesize more melatonin at night. However, in dystrophic RCS rats, the levels of pAANAT in photoreceptor cells is high during both daytime and nighttime (RCS_P20D vs. CTR_P20D
$P=0.049$, RCS_P35D vs. CTR_P35D $P=0.012$; Figure 2C), suggesting that photoreceptors synthesize high levels of melatonin during the day. Thus, rod-cone gap junction coupling is regulated by melatonin in a circadian clock-dependent manner. 


\section{The Signaling Pathway Underlying Rod-Cone Gap Junction Coupling}

Two melatonin receptors (MTs) are expressed in the rat retina, namely MT1 and MT2(Fujieda et al., 1999; Sallinen et al., 2005). We performed pharmacological experiments to identify the signaling cascade by which melatonin affects gap junction coupling. Applying the MT agonists Melatonin (Mel) and 2-Iodomelatonin (Iodo) (an MT1/2 agonist) resulted in significantly higher levels of gap junction coupling in the control animals in all age groups (P14, P20, and P35) during the day (Figures 3A,C,E). In dystrophic RCS rats, the results were more complicated. Mel and Iodo had no effect on P14 dystrophic RCS rats but decreased gap junction coupling at P20 (Figures 3A,C). At P35, Mel further increased gap junction coupling, but Iodo did not (Figure 3E). Hence, during the day, MTs are not activated in control animals but are activated in dystrophic RCS rats, indicating that melatonin levels are higher in the dystrophic RCS rat than in the control animals. The MT1/2 antagonist Luz dramatically decreased gap junction coupling at night in both groups of animals (Figures 3B,D,F). The MT antagonist 4-P-PDOT, which is $>300$-fold more selective for MT2, had almost no effect on gap junction coupling, suggesting that MT1 is the predominant receptor involved in regulating gap junction coupling. In many species, MT1 is expressed in a variety of retinal cells, including horizontal cells, glycinergic amacrine cells, dopaminergic amacrine cells, and some ganglion cells (Savaskan et al., 2002; Sallinen et al., 2005; Sengupta et al., 2011). Rodcone gap junction coupling is inhibited by dopamine, which is secreted from dopaminergic amacrine cells in a circadian fashion (Krizaj et al., 1998; Kothmann et al., 2011; Li et al., 2011; Choi et al., 2012; Jin et al., 2015). Thus, at night, increased extracellular melatonin binds the MT1 of dopaminergic amacrine cells to inhibit the synthesis and secretion of dopamine (Nguyen-Legros et al., 1996). Moreover, Ribelayga et al. (Ribelayga et al., 2008; Choi et al., 2012) demonstrated that the circadian clock in the retina decreases rod-cone gap junction coupling in the day by activating D2-like receptors. Taken together, these results indicate that in control animals at night and in dystrophic RCS rats at all

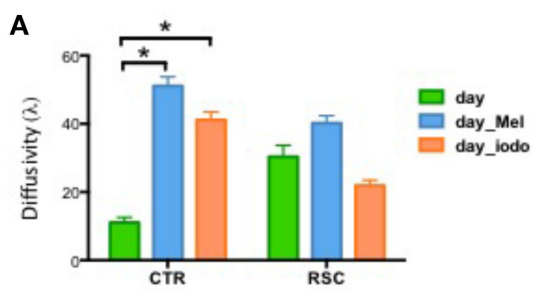

MT ant-/agonist in P14 to day $(n=6)$

C

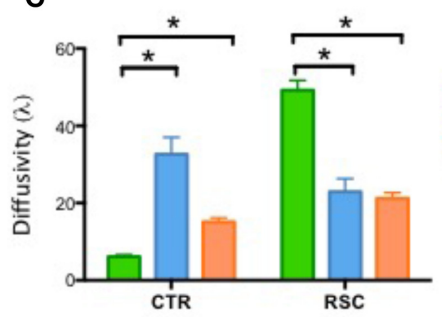

MT ant-/agonist in P20 to day $(n=6)$

E

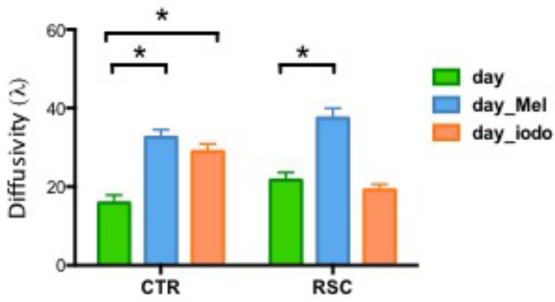

MT ant-/agonist in P35 to day $(n=6)$
B

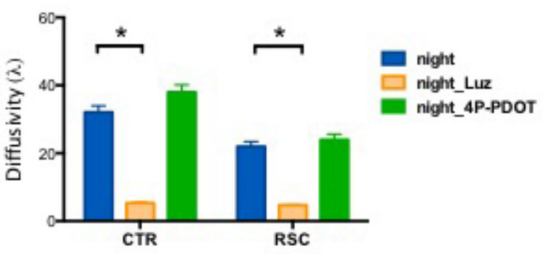

MT ant-/agonist in P14 to night $(n=6)$

D

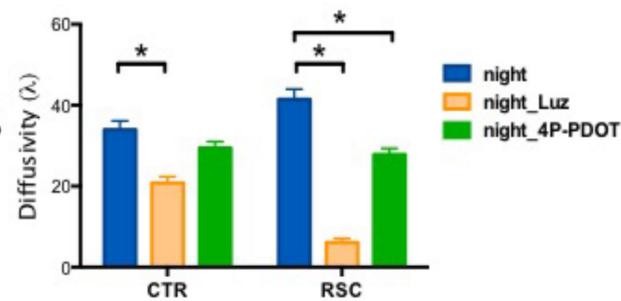

MT ant-/agonist in P20 to night $(n=6)$

$\mathbf{F}$

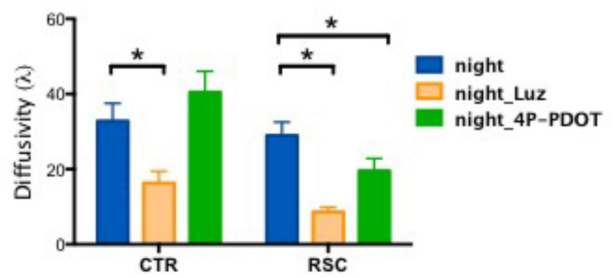

MT ant-/agonist in P35 to night $(n=6)$

FIGURE 3 | Changes in retinal diffusivity $(\lambda)$ in response to treatment with MT agonists or antagonists. During the daytime, lodo, and Mel increased gap junction coupling in the control groups at P14, P20, and P35 (A,C,E) but had the opposite effect in the RCS group at P20 (C). In RCS rats, lodo and Mel did not affect gap junction coupling at P14 (A), and Mel increased diffusivity at P35d (E). At night, Luz reduced diffusivity in both the control and RCS groups at P14, P20, and P35 (B,D,F), and 4-P-PDOT decreased the coupling ratio in RCS rats at P20 and P35 (D,F) but not at P14 (B). Mel, melatonin; lodo, 2-lodomelatonin (agonist of melatonin receptors); Luz, Luzindole (antagonist of melatonin receptors); 4-P-PDOT, Melatonin receptor 2 antagonist. ${ }^{*} P \leq 0.05$. 
times, the circadian clock modulates an increase in extracellular melatonin, which activates MT1 on dopaminergic cells. This leads to a decrease in the release of dopamine, resulting in the inactivation of the D2-like receptors on photoreceptors and a consequent increase in gap junction coupling.

\section{In Dystrophic RCS Rats, Daytime ERG Showed Partial Compensation by Rod-Cone Gap Junction Coupling}

To determine whether rod-cone coupling alters retinal visual functions, ERG recordings were conducted in both control and RCS rats during the daytime and nighttime (Figure 4). The a-wave of the ERG results from the photoreceptor component absorbing light, while the b-wave is generated mainly by bipolar cells and represents the secondary visual signal that occurs after input from photoreceptors (Gurevich and Slaughter, 1993; Robson and Frishman, 1998, 2004; Green and KapoustaBruneau, 1999). The amplitudes of both the a-wave and the bwave were smaller in dystrophic RCS rats than in the control group at ages P20 and P35 because of the loss of photoreceptors that was caused by apoptosis (Figure 4, Supplement Figure 4A). This finding is in agreement with the results of a previous study that used a similar approach in mice (Sengupta et al., 2011). In contrast, the dystrophic RCS rats showed weakened circadian regulation in the scotopic ERGs (Figures 4B,D,F; twoway ANOVA, $P>0.05)$. We averaged the ERG data from eight eyes obtained from four animals in each group and then extracted the mean responses for further analysis.

In the control group, there was no difference in the a-wave between day and night on P20 (Figure 4C), but there was a clear difference in the P35 group (Figures 4A,E), indicating circadian regulation. The $V_{\max }$ was larger at night than during the day $(424.03 \pm 0.0831$ vs. $388.99 \pm 18.9)$, whereas the $S$ was bigger $(0.5813 \pm 0.0831$ vs. $0.3444 \pm 0.0706$; Supplementary Table). These findings are consistent with the results of previous studies that have shown that cone responses are significantly smaller in amplitude at night. Thus, we conclude that in normal rats, ERGs can be used to monitor visual functions that are regulated by the circadian clock. In contrast, in dystrophic RCS rats, in the P20 and P35 groups, the $V_{\max }$ of the a-wave was similar between the day and night, whereas $S$ was somewhat larger at night than during the day (Supplementary Table). These results indicated that rod-cone gap junctions are functionally open both at night and during the day. There was a more pronounced difference in b-waves between day and night. In control rats, b-wave amplitude and velocity were dramatically different between daytime and nighttime in both age groups (amplitude, P20: day $595.08 \pm 48$ vs. night $722.78 \pm 36.6$ and P35: day $755 \pm 48.8$ vs. night $1020.9 \pm 23.7$; S, P20: day $0.0040 \pm$ 0.00197 vs. night $0.0029 \pm 0.0010$ and P35: day $0.0030 \pm 0.0012$ vs. night $0.0015 \pm 0.0000$; Supplementary Table). These data demonstrate that rod-cone gap junctions are functionally open at night but not during the day and that visual functions are continuing to develop at these ages. The thresholds for the aand $b$-wave in each group were calculated by fitting the responseillumination intensity with a hyperbolic function because the typical threshold measurement was inapplicable in dystrophic RCS rats, in which the b-wave has a lower threshold than the a-wave. In both control and dystrophic RCS rats, the b-wave appeared $\sim 2 \log$ units earlier than the a-wave (Figures 4D,F), but the amplitude of the b-wave was significantly smaller in dystrophic RCS rats than in control animals. In the dystrophic RCS rats, the b-wave amplitude was smaller at P35 than at P20, similar to the a-wave due to photoreceptor loss (Supplement Figures $4 \mathrm{~A}, \mathrm{~B})$. To further compare the kinetics of the a- and bwaves across various groups, the $\mathrm{a}$ - and $\mathrm{b}$-waves were normalized and fitted using a hyperbolic function (Fulton and Rushton, 1978; Hood and Birch, 1992).

The a-wave reflects photoreceptor activity, while the bwave originates from post-synaptic bipolar cells. Therefore, signal transmission in the distal retina can be transformed into the relationship between the b-wave and the a-wave. The b/a-ratio partially reflects the efficiency of signal transmission from the photoreceptors to bipolar cells. The amplitude of the a-wave grows at a faster rate than that of the b-wave, resulting in smaller b/a-ratios as intensity increases. Here, we refer to this phenomenon as e-gain. In this case, the b/aratio represents pure signal transmission efficiency because photoreceptor loss results in low amplitude a- and b-waves. The $\mathrm{b} / \mathrm{a}$-ratio therefore represents pure signal transmission efficiency and is not influenced by this side-effect. Thus, the b/a-ratio for each group was calculated using the fitting parameters for the individual group and was strongly dependent upon stimulus intensity. In control rats, e-gain was larger at night than during the day, a result that was confirmed by the b/awave ratio that was calculated from the raw data (Supplement Figure 3). Moreover, e-gain seemed to increase from P20 to P35, reflecting the functional maturation occurring in the connections between photoreceptors and bipolar cells during this period (Figure 5, Supplement Figure 3). This finding is also applicable to dystrophic RCS rats. Comparing the different age groups, $e$-gain values were higher in the dystrophic RCS rats in both age groups than in the comparable control groups, especially during in the daytime (Figure 5, Supplement Figures 3, 4C). Taken together, these data indicate that in dystrophic RCS rats, an increase in e-gain (b/a-wave ratio) compensates, at least partially, for the loss of visual input to bipolar cells that is caused by the loss of rods during early degenerative stages in these mice.

\section{DISCUSSION}

It has been previously demonstrated that rod-cone gap junctions open at night but not during the day in many animals, including goldfish and mice (Völgyi et al., 2013; Jin and Ribelayga, 2016). Patch-clamp data have shown that at night, dark-adapted cones can respond to light in the scotopic range because of the opening of rod-cone gap junctions (Szikra et al., 2014). Our data reveal that rats possess a mechanism of rod-cone gap junction coupling that is similar to that observed in other species (Asteriti et al., 2014). Furthermore, in the dystrophic RCS rat, which undergoes retinal degeneration, rod-cone gap junctions remain open during 
A

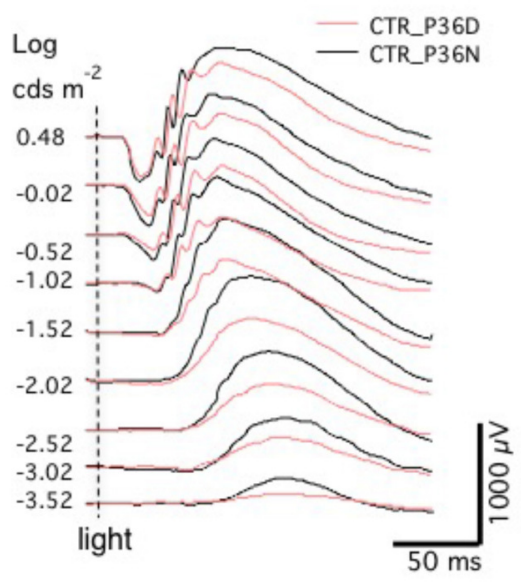

C

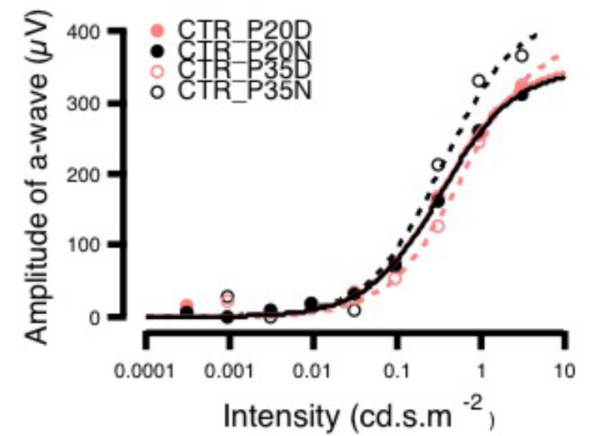

$\mathbf{E}$

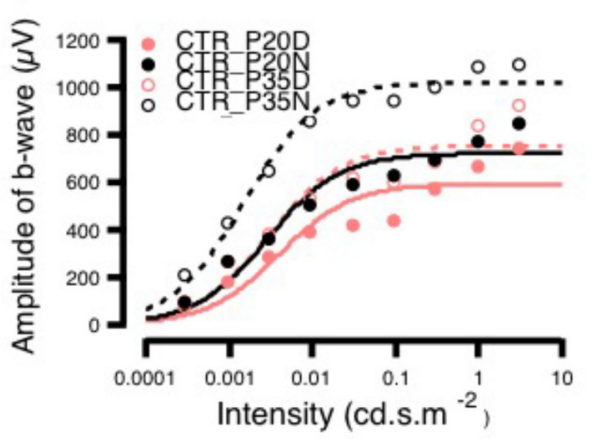

B

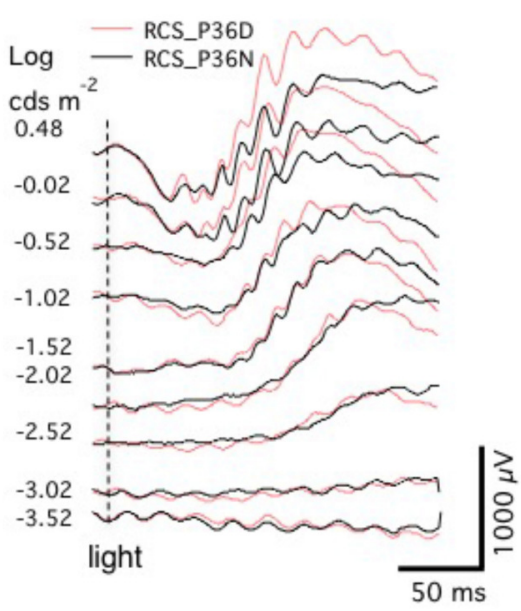

D

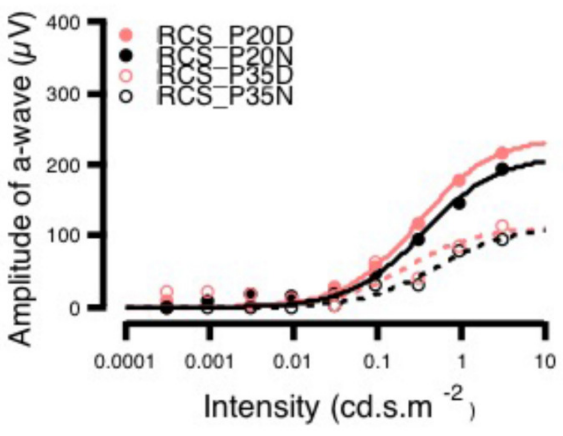

$\mathbf{F}$

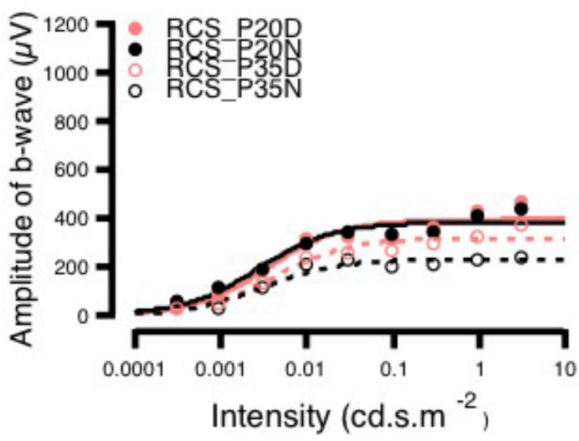

FIGURE 4 | Day-night differences in ERG a- and b-waves in rats. (A,B) Differences in ERG amplitudes between daytime and nighttime at P35 in control and $\mathrm{RCS}$ rats exposed to different light intensities. In the control group, at P35, there was no difference in the amplitude of the a-wave between daytime and nighttime when animals were exposed to a lower-intensity light stimulus, but when a higher luminance intensity was applied during the night, a-wave amplitudes sharply increased. The amplitudes of b-waves were increased more by low than by high light intensities. In the RCS group, the a-wave was not markedly different between the different light intensities, but b-wave amplitudes were higher in response to higher light intensities. In the control group, the amplitudes of a-waves increased with increasing light intensity at both P20 and P35. In the controls, at P20, the amplitude of a-waves was not clearly different between daytime and nighttime in response to different stimulus intensities. In animals exposed to medium light intensities, the amplitudes of a-waves were lower in the P35 daytime group than in the P20 daytime or nighttime groups and, and in animals exposed to either medium or high intensity light, the amplitudes were higher in the P35 nighttime group than in the P20 daytime and nighttime groups (C). In dystrophic RCS rats, at both P20 and P35, the amplitudes of a-waves increased with light intensity. The amplitudes of a-waves during the day in response to medium and high stimulus intensities were much lower at P35 than at P20. In addition, in the P35 RCS group, there were higher a-wave amplitudes during the daytime than at night (D). At both P20 and P35, the amplitudes of b-waves increased with light intensity. The b-waves had higher amplitudes at P35 than at P20 for each luminance intensity (E). The amplitudes of b-waves increased slightly with light intensity at both P20 and P35 in the control rats, and b-wave amplitudes were lower at P35 than at P20 for each light intensity (F).

the day at a level that is normally observed only at night. Interestingly, although the expression of the AANAT mRNA is regulated by the circadian clock (Tosini et al., 2006), we found that there was no difference in its protein level according to time of day, but there was a difference in the levels of its phosphorylated form between night and day. 


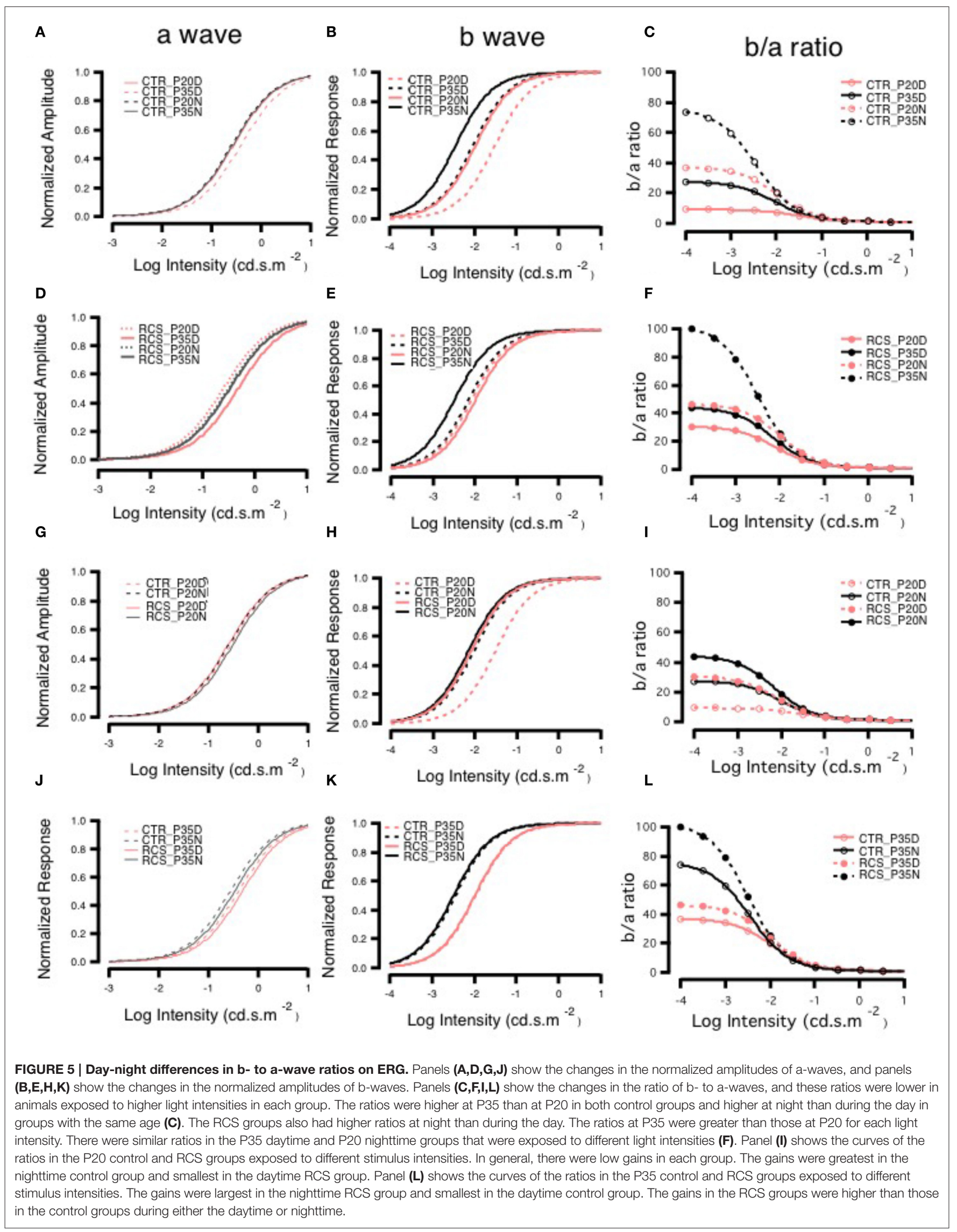


We used full-field flash ERG to determine whether gross retinal function is regulated by a circadian clock in these animals (Sengupta et al., 2011; Iuvone, 2014). Despite the fact that it is less sensitive than patch clamp, ERG can be used to monitor functional changes that are regulated by circadian clocks in control animals (Huang et al., 2012). The functional change in the a-wave is less pronounced than that in the b-wave. The b/a-ratio was calculated using raw data and fitting parameters (Sergeev et al., 2010; Ding et al., 2011; Young et al., 2012). Interestingly, in control rats, the b/a-ratio was greater at night than during the day and increased from P20 to P35. These data suggest that the amplitude of the b-wave (mainly reflecting signaling from bipolar cells) is more sensitive because the synaptic strength or efficiency of signaling between the photoreceptors and bipolar cells is regulated by a circadian clock (Emran et al., 2010; Lavoie et al., 2010; McMahon et al., 2014). The details of the mechanism underlying this phenomenon remains unknown. To our surprise, in both age groups, the b/a-ratio was larger in dystrophic RCS rats than in the control animals, although the amplitudes of the a- and b-waves were dramatically lower in dystrophic RCS rats at all intensities. While rod-cone coupling contributes to enhancing the signal of the first-order cells, there also seems to be a further effect from the synaptic connection between photoreceptor and bipolar cells and perhaps the signaling of the bipolar cell as well (Ribelayga et al., 2002).

Second, in both normal rats and dystrophic RCS rats, increased rod-cone gap junction coupling at night tunes the retina, allowing it to detect large, dimly lit objects (Tsukamoto et al., 2001; Ribelayga et al., 2008). During the day, because the number of rod cells decreases during P20-P35 in dystrophic RCS rats, the visual signal conveyed by cones can also propagate to rods via gap junction couplings. Thus, rods converge onto rod bipolar cells that also receive cone input, and this convergence increases the visual signal of bipolar cells. This homeostatic plasticity partially rescues the impaired visual functions caused by the loss of photoreceptor cells (Gargini et al., 2007; Strettoi et al., 2010).

Finally, cone survival may depend on the gap junctionmediated diffusion of nutrients and protective factors from healthy rods (Striedinger et al., 2005). In dystrophic RCS rats, rods die earlier than cones. In early stages, this process might be slowed by the diffusion of nutrients and protective factors from neighboring cones. However, cones might also die as a result of the diffusion of apoptotic factors from coupled dying rods (Ripps, 2002). When all rods are gone, the cones may die

\section{REFERENCES}

Asteriti, S., Gargini, C., and Cangiano, L. (2014). Mouse rods signal through gap junctions with cones. Elife Sci. 3, 162-171. doi: 10.7554/elife.01386

Choi, B. H., Chae, H. D., Park, T. J., Oh, J., Lim, J., Kang, S. S., et al. (2004). Protein kinase $\mathrm{C}$ regulates the activity and stability of serotonin $\mathrm{N}$-acetyltransferase. J. Neurochem. 90, 442-454. doi: 10.1111/j.1471-4159.2004.02495.x

Choi, H. J., Ribelayga, C. P., and Mangel, S. C. (2012). Cut-loading: a useful tool for examining the extent of gap junction tracer coupling between retinal neurons. J. Vis. Exp. 221-221. doi: 10.3791/3180 quickly as a result of the loss of support from rods. In addition, injecting melatonin increases light-induced damage to the retina, and this effect can be rescued by pretreatment with luzindole, a competitive antagonist of the melatonin receptor (Sugawara et al., 1998). The mechanism by which melatonin increases susceptibility to light damage remains unclear, but gap junction coupling is a potential explanation. In any case, higher levels of melatonin increase both susceptibility to light damage and the diffusion of apoptotic factors via gap junction couplings, and these processes in turn quicken the loss of photoreceptors.

In summary, the lifespan of cone cells is shortened because they both support rods via rod-cone gap junction couplings and act as sensors on their own. On the one hand, increasing rodcone gap junction coupling enhances visual signals, allowing rats to detect large, dim objects at night, and this process might also allow better vision during the day if the rod bipolar pathway from cones to rods is activated. On the other hand, the pathological increase in melatonin and constant presence of open rod-cone gap junction couplings collectively hasten the death of photoreceptors because they promote the diffusion of apoptotic factors through open gap junctions.

\section{AUTHOR CONTRIBUTIONS}

$\mathrm{BH}$ and YF contributed to the conception and design of the study, the collection and assembly of data, data analysis and interpretation, and manuscript writing; CW and WL contribute to the collection and assembly of data; CZ contributed to the conception and design of the study, data analysis and interpretation, manuscript writing, and the final approval of manuscript; and ZY contributed to the conception and design of the study, its financial support, data analysis and interpretation, and the final approval of manuscript.

\section{FUNDING}

This work was supported by National Basic Research Program of China (973 Program, 2013CB967002), the National Natural Science Foundation Project 81130017, and 81371032.

\section{SUPPLEMENTARY MATERIAL}

The Supplementary Material for this article can be found online at: http://journal.frontiersin.org/article/10.3389/fncel. 2017.00098/full\#supplementary-material transmission. BMC Cell Biol. 17(Suppl. 1):13. doi: 10.1186/s12860-016-0091-y

Day, A. C., Rotsos, T., Holder, G. E., Tufail, A., and Robson, A. G. (2010). Electrodiagnostic and two-wavelength fundus autofluorescence imaging investigations in acute idiopathic maculopathy. Doc. Ophthalmol. 121, 155-160. doi: 10.1007/s10633-010-9235-0

Ding, J. D., Johnson, L. V., Herrmann, R., Farsiu, S., Smith, S. G., Kelly, U., et al. (2011). Anti-amyloid therapy protects against retinal pigmented epithelium damage and vision loss in a model of age-related macular degeneration. Proc. Natl. Acad. Sci. U.S.A. 108, 279-287. doi: 10.1073/pnas.1100901108 
D’Orazi, F. D., Suzuki, S. C., and Wong, R. O. (2014). Neuronal remodeling in retinal circuit assembly, disassembly, and reassembly. Trends Neurosci. 37, 594-603. doi: 10.1016/j.tins.2014.07.009

Emran, F., Rihel, J., Adolph, A. R., and Dowling, J. E. (2010). Zebrafish larvae lose vision at night. Proc. Natl. Acad. Sci. U.S.A. 107, 6034-6039. doi: 10.1073/pnas.0914718107

Fujieda, H., Hamadanizadeh, S. A., Wankiewicz, E., Pang, S. F., and Brown, G. M. (1999). Expression of $\mathrm{mt}_{1}$ melatonin receptor in rat retina: evidence for multiple cell targets for melatonin. Neuroscience 93, 793-799. doi: 10.1016/S0306-4522(99)00111-6

Fulton, A. B., and Rushton, W. A. H. (1978). The human rod ERG: correlation with psychophysical responses in light and dark adaptation. Vision Res. 18, 793-800.

Gargini, C., Terzibasi, E., Mazzoni, F., and Strettoi, E. (2007). Retinal organization in the retinal degeneration 10 (rd10) mutant mouse: a morphological and ERG study. J. Comp. Neurol. 500, 222-238. doi: 10.1002/cne.21144

Girman, S. V., Wang, S., and Lund, R. D. (2005). Time course of deterioration of rod and cone function in RCS rat and the effects of subretinal cell grafting: a light- and dark-adaptation study. Vis. Res. 45, 343-354. doi: 10.1016/j.visres.2004.08.023

Green, D. G., and Kapousta-Bruneau, N. V. (1999). A dissection of the electroretinogram from the isolated rat retina with microelectrodes and drugs. Vis. Neurosci. 16, 727-741. doi: 10.1017/S0952523899164125

Guo, C. X., Tran, H., Green, C. R., Danesh-Meyer, H. V., and Acosta, M. L. (2014). Gap junction proteins in the light-damaged albino rat. Mol. Vis. 20, 670-682.

Gurevich, L., and Slaughter, M. M. (1993). Comparison of the waveforms of the ON bipolar neuron and the b-wave of the electroretinogram. Vis. Res. 33, 2431-2435. doi: 10.1016/0042-6989(93)90122-D

Hiragaki, S., Baba, K., Coulson, E., Kunst, S., Spessert, R., and Tosini, G. (2014). Melatonin signaling modulates clock genes expression in the mouse retina. PLoS ONE 9:e106819. doi: 10.1371/journal.pone.0106819

Hood, D. C., and Birch, D. G. (1992). A computational model of the amplitude and implicit time of the b-wave of the human ERG. Vis. Neurosci. 8, 107-126.

Huang, H., Wang, Z., Weng, S. J., Sun, X. H., and Yang, X. L. (2012). Neuromodulatory role of melatonin in retinal information processing. Prog. Retin. Eye Res. 32, 64-87. doi: 10.1016/j.preteyeres.2012.07.003

Iuvone, P. M. (2014). The Retina and Circadian Rhythms. New York, NY: Springer.

Jin, N. G., and Ribelayga, C. P. (2016). Direct evidence for daily plasticity of electrical coupling between rod photoreceptors in the mammalian retina. J. Neurosci. 36, 178-184. doi: 10.1523/JNEUROSCI.3301-15.2016

Jin, N. G., Chuang, A. Z., Masson, P. J., and Ribelayga, C. P. (2015). Rod electrical coupling is controlled by a circadian clock and dopamine in mouse retina. J. Physiol. 593, 1597-1631. doi: 10.1113/jphysiol.2014.284919

Kashiwagi, T., Park, Y. J., Park, J. G., Imamura, S., Takeuchi, Y., Hur, S. P., et al. (2013). Moonlight affects mRNA abundance of arylalkylamine $\mathrm{N}$-acetyltransferase in the retina of a lunar-synchronized spawner, the goldlined spinefoot. J. Exp. Zool. A Ecol. Genet. Physiol. 319, 505-516. doi: $10.1002 /$ jez.1814

Kothmann, W. W., Massey, S. C., and O’Brien, J. (2011). Dopamine-stimulated dephosphorylation of connexin 36 mediates AII amacrine cell uncoupling. J. Neurosci. 29, 14903-14911. doi: 10.1523/JNEUROSCI.3436-09.2009

Krizaj, D., Gábriel, R., Owen, W. G., and Witkovsky, P. (1998). Dopamine D2 receptor-mediated modulation of rod-cone coupling in the Xenopus retina. J. Comp. Neurol. 398, 529-538. doi: 10.1002/(SICI)1096-9861(19980907) 398:4<529::AID-CNE5>3.0.CO;2-4

Lavoie, J., Gagné, A. M., Lavoie, M. P., Sasseville, A., Charron, M. C., and Hébert, M. (2010). Circadian variation in the electroretinogram and the presence of central melatonin. Doc. Ophthalmol. 120, 265-272. doi: 10.1007/s10633-010-9221-6

Li, H., Chuang, A. Z., and O'Brien, J. (2014). Regulation of photoreceptor gap junction phosphorylation by adenosine in zebrafish retina. Vis. Neurosci. 31, 237. doi: 10.1017/s095252381300062x

Li, H., Zhang, Z., Blackburn, M. R., Wang, S. W., Ribelayga, C. P., and O’Brien, J. (2011). Adenosine and dopamine receptors coregulate photoreceptor coupling via gap junction phosphorylation in mouse retina. J. Neurosci. 33, 3135-3150. doi: 10.1523/JNEUROSCI.2807-12.2013

McMahon, D. G., Iuvone, P. M., and Tosini, G. (2014). Circadian organization of the mammalian retina: from gene regulation to physiology and diseases. Prog. Retin. Eye Res. 39, 58-76. doi: 10.1016/j.preteyeres.2013.12.001
Nguyen-Legros, J., Chanut, E., Versaux-Botteri, C., Simon, A., and Trouvin, J. H. (1996). Dopamine inhibits melatonin synthesis in photoreceptor cells through a D2-like receptor subtype in the rat retina: biochemical and histochemical evidence. J. Neurochem. 67, 2514-2520. doi: 10.1046/j.1471-4159.1996.67062514.x

Pozdeyev, N., Taylor, C., Haque, R., Chaurasia, S. S., Visser, A., Thazyeen, A., et al. (2006). Photic regulation of arylalkylamine N-acetyltransferase binding to 14-3-3 proteins in retinal photoreceptor cells. J. Neurosci. 26, 9153-9161. doi: 10.1523/JNEUROSCI.1384-06.2006

Ribelayga, C., Cao, Y., and Mangel, S. C. (2008). The circadian clock in the retina controls rod-cone coupling. Neuron 59, 790-801. doi: 10.1016/j.neuron. 2008.07.017

Ribelayga, C., Wang, Y., and Mangel, S. C. (2002). Dopamine mediates circadian clock regulation of rod and cone input to fish retinal horizontal cells. J. Physiol. 544, 801-816. doi: 10.1113/jphysiol.2002.023671

Ripps, H. (2002). Cell death in retinitis pigmentosa: gap junctions and the 'bystander' effect. Exp. Eye Res. 74, 327-336. doi: 10.1006/exer.2002.1155

Robson, J. G., and Frishman, L. J. (1998). Dissecting the dark-adapted electroretinogram. Doc. Ophthalmol. 95, 187-215. doi: 10.1023/A:100 1891904176

Robson, J. G., and Frishman, L. J. (2004). Sampling and interpolation of the a-wave of the electroretinogram. Doc. Ophthalmol. 108, 171-179. doi: $10.1007 /$ s10633-004-4443-0

Sakamoto, K., and Ishida, N. (1998). Circadian expression of serotonin $\mathrm{N}$-acetyltransferase mRNA in the rat retina. Neurosci. Lett. 245, 113-116. doi: 10.1016/S0304-3940(98)00189-X

Sallinen, P., Saarela, S., Ilves, M., Vakkuri, O., and Leppäluoto, J. (2005). The expression of MT1 and MT2 melatonin receptor mRNA in several rat tissues. Life Sci. 76, 1123-1134. doi: 10.1016/j.lfs.2004.08.016

Sandu, C., Hicks, D., and Felder-Schmittbuhl, M. P. (2011). Rat photoreceptor circadian oscillator strongly relies on lighting conditions. Eur. J. Neurosci. 34, 507-516. doi: 10.1111/j.1460-9568.2011.07772.x

Sauvé, Y., Girman, S. V., Wang, S., Lawrence, J. M., and Lund, R. D. (2001). Progressive visual sensitivity loss in the Royal College of Surgeons rat: perimetric study in the superior colliculus. Neuroscience 103, 51-63. doi: 10.1016/S0306-4522(00)00557-1

Savaskan, E., Wirzjustice, A., Olivieri, G., Pache, M., Kräuchi, K., Brydon, L., et al. (2002). Distribution of melatonin MT1 receptor immunoreactivity in human retina. J. Histochem. Cytochem. 50, 519-526. doi: 10.1177/0022155402050 00408

Sengupta, A., Baba, K., Mazzoni, F., Pozdeyev, N. V., Strettoi, E., Iuvone, P. M., et al. (2011). Localization of melatonin receptor 1 in mouse retina and its role in the circadian regulation of the electroretinogram and dopamine levels. PLoS ONE 6:e0024483. doi: 10.1371/journal.pone.0024483

Sergeev, Y. V., Caruso, R. C., Meltzer, M. R., Smaoui, N., MacDonald, I. M., and Sieving, P. A. (2010). Molecular modeling of retinoschisin with functional analysis of pathogenic mutations from human X-linked retinoschisis. Hum. Mol. Genet. 19, 1302-1313. doi: 10.1093/hmg/ddq006

Strettoi, E., Gargini, C., Novelli, E., Sala, G., Piano, I., Gasco, P., et al. (2010). Inhibition of ceramide biosynthesis preserves photoreceptor structure and function in a mouse model of retinitis pigmentosa. Proc. Natl. Acad. Sci. U.S.A. 107, 18706-18711. doi: 10.1073/pnas.1007644107

Striedinger, K., Petrasch-Parwez, E., Zoidl, G., Napirei, M., Meier, C., Eysel, U. T., et al. (2005). Loss of connexin 36 increases retinal cell vulnerability to secondary cell loss. Eur. J. Neurosci. 22, 605-616. doi: 10.1111/j.1460-9568.2005. 04228.x

Sugawara, T., Sieving, P. A., Iuvone, P. M., and Bush, R. A. (1998). The melatonin antagonist luzindole protects retinal photoreceptors from light damage in the rat. Invest. Ophthalmol. Vis. Sci. 39, 2458-2465.

Szikra, T., Trenholm, S., Drinnenberg, A., Jüttner, J., Raics, Z., Farrow, $\mathrm{K}$, et al. (2014). Rods in daylight act as relay cells for cone-driven horizontal cell-mediated surround inhibition. Nat. Neurosci. 17, 1728-1735. doi: $10.1038 / \mathrm{nn} .3852$

Tosini, G., Chaurasia, S. S., and Michael Iuvone, P. (2006). Regulation of Arylalkylamine $N$-Acetyltransferase (AANAT) in the Retina. Chronobiol. Int. 23, 381-391. doi: 10.1080/07420520500482066

Tsukamoto, Y., Morigiwa, K., Ueda, M., and Sterling, P. (2001). Microcircuits for night vision in mouse retina. J. Neurosci. 21, 8616-8623. 
Van Den Berghe, L., Sainton, K. K., Marchant, D., Dufour, E., Bonnel, S., Gadin, S., et al. (2004). Prosaposin gene expression in normal and dystrophic RCS rat retina. Invest. Ophthalmol. Vis. Sci. 45, 1297-1305. doi: 10.1167/iovs. 03-1048

Völgyi, B., Kovács-Öller, T., Atlasz, T., Wilhelm, M., and Gábriel, R. (2013). Gap junctional coupling in the vertebrate retina: variations on one theme? Prog. Retin. Eye Res. 34, 1-18. doi: 10.1016/j.preteyeres.2012. 12.002

Weymouth, A. E., and Vingrys, A. J. (2008). Rodent electroretinography: methods for extraction and interpretation of rod and cone responses. Prog. Retin. Eye Res. 27, 1-44. doi: 10.1016/j.preteyeres.2007.09.003

Young, B., Eggenberger, E., and Kaufman, D. (2012). Current electrophysiology in ophthalmology: a review. Curr. Opin. Ophthalmol. 23, 497-505. doi: 10.1097/ICU.0b013e328359045e
Zhang, J., and Wu, S. M. (2004). Connexin35/36 gap junction proteins are expressed in photoreceptors of the tiger salamander retina. J. Comp. Neurol. 470, 1-12. doi: 10.1002/cne.10967

Conflict of Interest Statement: The authors declare that the research was conducted in the absence of any commercial or financial relationships that could be construed as a potential conflict of interest.

Copyright $\odot 2017 \mathrm{Hou}, \mathrm{Fu}$, Weng, Liu, Zhao and Yin. This is an open-access article distributed under the terms of the Creative Commons Attribution License (CC BY). The use, distribution or reproduction in other forums is permitted, provided the original author(s) or licensor are credited and that the original publication in this journal is cited, in accordance with accepted academic practice. No use, distribution or reproduction is permitted which does not comply with these terms. 